impact on the immunogenicity of HIV antigens vectored in rare serotype human adenoviral vectors? Frahm et al. did not have samples from such clinical trials available to them to test this hypothesis. However, clinical trials employing these vectors have started, and more will follow soon, which will allow for the hypothesis to be tested in the context of rigorous clinical investigation, thus providing critical insight to guide the development of a globally effective HIV vaccine.

\section{Acknowledgments}

The views expressed here belong solely to the author and are not to be construed as reflecting the views of the Department of the Army or the Department of Defense.

Address correspondence to: Nelson L. Michael, US Military HIV Research Program, Walter Reed Army Institute of Research, 6720-B Rockledge Drive, Suite 400, Bethesda, Maryland 20817, USA. Phone: 301.500.3601; Fax: 301.500.3666; E-mail: nmichael@hivresearch.org.

1. UNAIDS. Global Report: UNAIDS Report of the Global AIDS Epidemic. Geneva, Switzerland: Joint United Nations Program on HIV/AIDS (UNAIDS); 2010. 2. Auvert B, Taljaard D, Lagarde E, Sobngwi-Tam- bekou J, Sitta R, Puren A. Randomized, controlled intervention trial of male circumcision for reduction of HIV infection risk: the ANRS 1265 Trial. PLoS Med. 2005;2(11):e298.

3. Bailey RC, et al. Male circumcision for HIV prevention in young men in Kisumu, Kenya: a randomised controlled trial. Lancet. 2007; 369(9562):643-656.

4. Gray RH, et al. The impact of male circumcision on HIV incidence and cost per infection prevented: a stochastic simulation model from Rakai, Uganda. AIDS. 2007;21(7):845-850.

5. Abdool Karim Q, et al. Effectiveness and safety of tenofovir gel, an antiretroviral microbicide, for the prevention of HIV infection in women. Science. 2010;329(5996):1168-1174.

6. Grant RM, et al. Preexposure chemoprophylaxis for HIV prevention in men who have sex with men. N Engl J Med. 2010;363(27):2587-2599.

7. Cohen MS, et al. Prevention of HIV-1 infection with early antiretroviral therapy. $N$ Engl J Med. 2011;365(6):493-505.

8. Johnston MI, Fauci AS. HIV vaccine developmentimproving on natural immunity. NEnglJ Med. 2011; 365(10):873-875.

9. Buchbinder SP, et al. Efficacy assessment of a cellmediated immunity HIV-1 vaccine (the Step Study): a double-blind, randomised, placebo-controlled, testof-concept trial. Lancet. 2008;372(9653):1881-1893.

10. Flynn NM, Forthal DN, Harro CD, Judson FN, Mayer KH, Para MF. Placebo-controlled phase 3 trial of a recombinant glycoprotein 120 vaccine to prevent HIV-1 infection. J Infect Dis. 2005; 191(5):654-665.

11. Pitisuttithum P, et al. Randomized, double-blind, placebo-controlled efficacy trial of a bivalent recombinant glycoprotein $120 \mathrm{HIV}-1$ vaccine among injection drug users in Bangkok, Thailand. J Infect Dis. 2006;194(12):1661-1671.
12. Rerks-Ngarm S, et al. Vaccination with ALVAC and AIDSVAX to prevent HIV-1 infection in Thailand. N Engl J Med. 2009;361(23):2209-2220.

13. Duerr AC, Huang Y, Moodie Z, Lawrence D, Robertson D, Buchbinder S. Analysis of the relative risk of HIV acquisition among Step study participants with extended follow-up. Presented at: AIDS Vaccine Conference; September 28-October 1, 2010; Atlanta, Georgia, USA.

14. Kim JH, Rerks-Ngarm S, Excler JL, Michael NL. HIV vaccines: lessons learned and the way forward. Curr Opin HIV AIDS. 2010;5(5):428-434.

15. Cheng $C$, et al. Differential specificity and immunogenicity of adenovirus type 5 neutralizing antibodies elicited by natural infection or immunization. J Virol. 2010;84(1):630-638.

16. Abbink P, et al. Comparative seroprevalence and immunogenicity of six rare serotype recombinant adenovirus vaccine vectors from subgroups $B$ and $D$. J Virol. 2007;81(9):4654-4663.

17. Mast TC, et al. International epidemiology of human pre-existing adenovirus (Ad) type-5, type-6, type- 26 and type-36 neutralizing antibodies: correlates of high Ad5 titers and implications for potential HIV vaccine trials. Vaccine. 2010;28(4):950-957.

18. Frahm N, et al. Human adenovirus-specific $T$ cells modulate HIV-specific T cell responses to an Ad5-vectored HIV-1 vaccine. J Clin Invest. 2012; 122(1):359-367.

19. Liu J, et al. Immune control of an SIV challenge by a T-cell-based vaccine in rhesus monkeys. Nature. 2009; 457(7225):87-91.

20. Farina SF, et al. Replication-defective vector based on a chimpanzee adenovirus. J Virol. 2001; 75(23):11603-11613.

21. Kong WP, et al. Expanded breadth of the T-cell response to mosaic human immunodeficiency virus type 1 envelope DNA vaccination.J Virol. 2009; 83(5):2201-2215

\title{
IL-1 and atherosclerosis: a murine twist to an evolving human story
}

\author{
Daniel J. Rader
}

Department of Medicine and Institute for Translational Medicine and Therapeutics, Perelman School of Medicine at the University of Pennsylvania, Philadelphia, Pennsylvania, USA.

\begin{abstract}
Inflammation is a critical component of atherosclerosis. IL-1 is a classic proinflammatory cytokine that has been linked to atherosclerosis. A clinical trial has been launched in which an antibody specific for IL-1 $\beta$ is being studied for its effects on cardiovascular events in patients with atherosclerosis. In this issue of the JCI, Alexander et al. report that mice lacking the receptor for IL-1 unexpectedly have features of advanced atherosclerosis that suggest the atherosclerotic plaques may be less stable. These findings illustrate the complexity of inflammatory pathways in atherosclerosis and suggest the need for careful calibration of antiinflammatory approaches to atherosclerosis.
\end{abstract}

Coronary artery disease (CAD) is the leading cause of death in the United States for both men and women. CAD is caused by

Conflict of interest: Daniel J. Rader declares a conflict of interest with Aegerion, Merck, Novartis, and Pfizer.

Citation for this article: J Clin Invest. 2012;

122(1):27-30. doi:10.1172/JCI61163. the progressive formation in the coronary arteries of atherosclerotic plaques that are characterized by the accumulation of lipids, in particular cholesterol and its derivatives, and inflammatory cells. In the early stages of disease development, the atherosclerotic plaques are small, lipid rich, and asymptomatic. Over time, they mature into advanced atherosclerotic plaques, with increased content of vascular smooth muscle cells, extracellular matrix, and inflammatory markers, and gain characteristics that may have clinical consequences. Clinical symptoms can arise from plaques causing flow-limiting stenoses, although the process of compensatory remodeling involving expansion of the vessel can help to protect against this. The more clinically important consequence of coronary atherosclerosis is the rupture or disruption of a "vulnerable" plaque. Plaque rupture leads to prothrombotic material in the plaque becoming exposed to the blood and to thrombosis, resulting in acute coronary events, including myocardial infarction and death. There has been substantial 


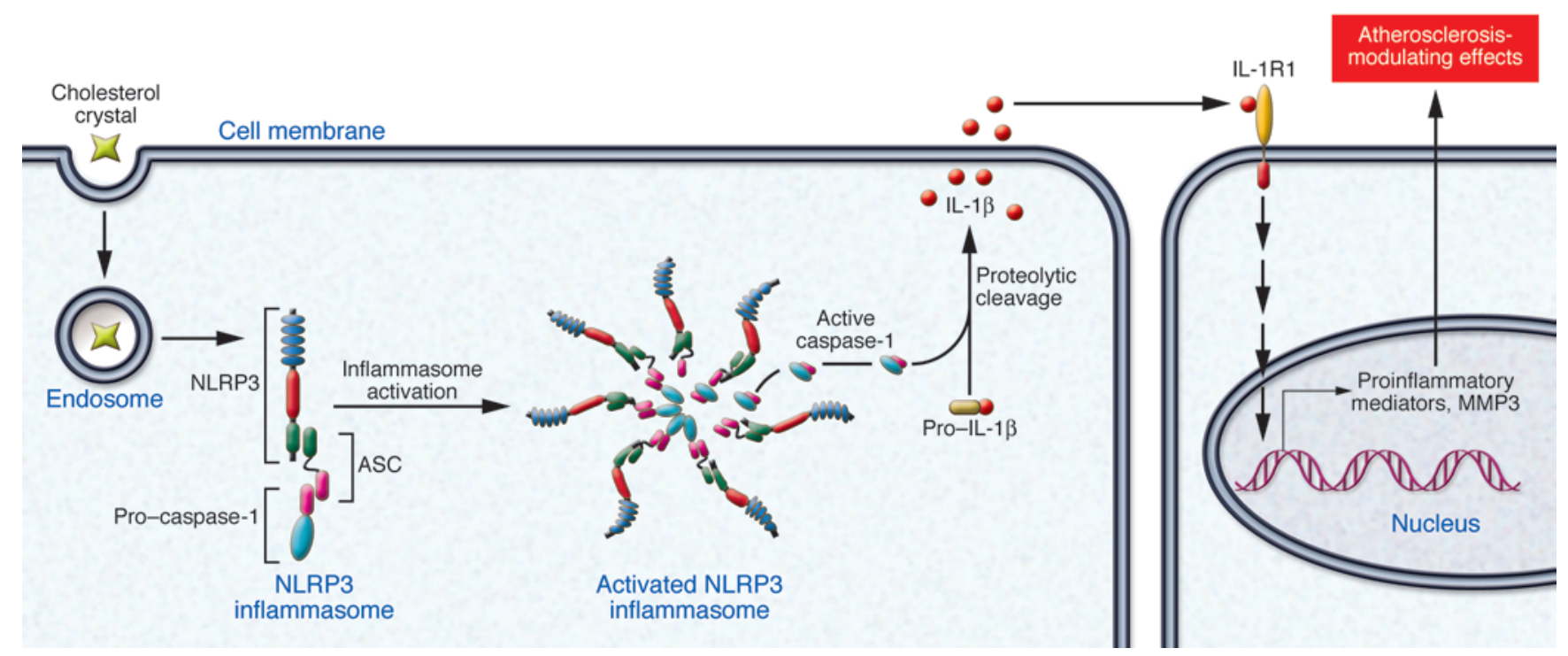

Figure 1

Role of the NLRP3 inflammasome and IL-1 $\beta$ in atherosclerosis. Extracellular cholesterol crystals act as a "danger signal" and activate the NLRP3 inflammasome, which triggers activation of caspase-1, leading to cleavage of pro-IL-1 $\beta$ to its active form. The active form of IL-1 $\beta$ is then secreted from the cell, where it mediates autocrine and paracrine effects through activation of the IL-1R1.

interest in identifying pathways that lead to plaque instability and in the development of novel therapies that stabilize the vulnerable plaque and reduce acute coronary events (1).

\section{Inflammation and atherosclerosis}

Inflammation plays a key role in all stages of atherogenesis, from recruitment of monocytes and other leukocytes to growth of the plaque to plaque rupture and thrombosis $(1,2)$. A variety of inflammatory pathways have been associated with human atherosclerosis, and such links have been investigated extensively in mouse models of atherogenesis (3). A remarkable number of inflammatory mediators - including a myriad of cytokines and their receptors, chemokines and their receptors, and intracellular signaling molecules - have been shown when deleted from the mouse genome to influence plaque size, often (but not always) in the direction of reduced atherosclerosis when the relevant inflammatory pathway is interrupted (3). However, the relevance of these studies to human atherosclerosis remains uncertain in the absence of direct interventional randomized controlled trials in humans.

There has been interest for several years in the potential role of the proinflammatory cytokine IL-1 in atherogenesis (4). Both IL- $1 \alpha$ and IL- $1 \beta$ activate IL- 1 receptor type I (IL-1R1), and IL-1 receptor antago- nist (IL-1ra) competitively inhibits their binding. IL-1 $\alpha$ remains associated with the plasma membrane, whereas IL- $1 \beta$ is fully secreted and can act on IL-1R1 on cells distant from the cell of origin. Thus IL- $1 \beta$ has been thought to be of greater relevance to human inflammatory diseases.

Recently there has been considerable interest in the inflammasome, a multimolecular cellular complex containing nucleotide-binding domain-, leucinerich repeat-, and pyrin domain-containing receptors (NLRPs). The inflammasome responds to endogenous danger signals by activating caspase-1, which in turn converts pro-IL-1 $\beta$ into the mature active form (Figure 1 and refs. 5, 6). Certain genetic mutations affecting proteins of the NLRP3 inflammasome cause rare autoinflammatory disorders characterized by excessive IL-1 $\beta$ production (e.g., Muckle-Wells syndrome and familial cold autoinflammatory syndrome; ref. 7); IL-1 $\beta$ inhibition appears to be efficacious in these settings (8). More common conditions are also characterized by activation of the NLRP3 inflammasome and excessive production of IL-1 $\beta$. For example, urate crystals in the joint activate NLRP3 and drive IL- $1 \beta$ production, leading to acute gout (9). Thioredoxin-interacting protein (TXNIP), expression of which is upregulated by glucose, is a direct ligand for and activates NLRP3 and thus promotes IL-1 $\beta$ production, possibly exacer- bating type 2 diabetes (10). Indeed, antiIL-1 therapies have been shown to have some efficacy in type 2 diabetes $(11,12)$.

Because atherosclerosis is a chronic inflammatory condition that IL- $1 \beta$ has been linked to in the past $(4,13)$, it was of high interest when, in 2010, two publications implicated the NLRP3 inflammasome in atherosclerosis by demonstrating that cholesterol crystals activate the NLRP3 inflammasome and generate IL-1 $\beta$ production (Figure 1 and refs. 14, 15). Atherosclerosis-prone mice reconstituted with bone marrow from NLRP3deficient or IL-1 $\beta$-deficient mice were shown to have less atherosclerosis (14), consistent with a causal role for this pathway in promoting atherogenesis. This observation garnered considerable interest because of a clear pathway linking the inflammasome to atherosclerosis and the implications for novel therapeutic approaches $(10,16,17)$.

\section{Anti-IL-1 $\beta$ therapy and acute coronary events: a clinical trial}

Spurred by the data linking the NLRP3 inflammasome/IL-1 $\beta$ pathway to atherogenesis $(12,13)$ and earlier work indicating a causal role for IL- $1 \beta$ in promoting atherosclerosis in mice $(18,19)$, an ambitious clinical trial was recently launched. Canakinumab is a humanized monoclonal antibody specific for IL- $1 \beta$ that is efficacious against and currently approved 
for autoinflammatory disorders driven by excess IL-1 $\beta$ (including Muckle-Wells syndrome and familial cold autoinflammatory syndrome) (8). The Canakinumab Anti-inflammatory Thrombosis Outcomes Study (CANTOS) is designed to test the hypothesis that IL- $1 \beta$ inhibition will reduce major cardiovascular events in persons with preexisting CAD (20). Because of uncertainty about the optimal degree of IL- $1 \beta$ inhibition needed to achieve cardiovascular event reduction with acceptable safety, canakinumab will be tested at three different doses compared with placebo, for a total of four parallel arms in a study planned to enroll more than 17,000 subjects. This trial is the first true test of the hypothesis that interrupting an inflammatory pathway involved in atherosclerosis will reduce cardiovascular events.

\section{IL-1 receptor deficiency and influence on advanced atherosclerosis in mice}

Given the initiation of the CANTOS trial, the paper by Alexander et al. in this issue of the JCI (21) is of considerable interest. The authors investigated the effects of deletion of the gene encoding IL-1R1 on advanced atherosclerotic plaques in atherosclerosisprone ApoE-deficient mice fed a high-fat diet for up to 30 weeks. Consistent with previous reports $(18,22)$ and the authors' a priori hypothesis, overall plaque size in the aortic root (the traditional location for assessment of mouse atherosclerosis) was reduced in mice lacking IL-1R1, supporting a role for IL-1 signaling in promoting plaque growth in the mouse (21). However, the authors also examined the brachiocephalic artery, another site of atherosclerosis in disease-prone mice. Unexpectedly, the authors found in the IL-1R1-deficient mice that plaque size was not reduced and, furthermore, that the atherosclerotic vessels had reduced lumen size as a result of reduced outward vessel remodeling. Upon examination of the aortic roots, the same observation of impaired outward remodeling was made. In addition, plaques at both sites demonstrated reduced vascular smooth muscle cell and collagen content and increased intraplaque hemorrhage, all markers associated with plaque instability on autopsy of human coronary arteries. Alexander and colleagues further provided data suggesting that IL-1 signaling increases expression of matrix metalloproteinase 3 (MMP3) and that relative deficiency of MMP3 in the IL-1R1-deficient animals may have contributed to the observed atherosclerotic vascular phenotypes. These results in mice could be interpreted to be consistent with a protective role of IL-1 signaling in advanced atherosclerotic plaques.

\section{Implications for IL-1 $\beta$ inhibition in humans}

Should the findings of Alexander et al. (21) lead to concern about the clinical trial of the IL-1 $\beta$-specific antibody canakinumab in subjects with CAD? There are a number of reasons to be cautious about extrapolating these results in mice to the implications of inhibiting IL-1 $\beta$ in humans. First, Alexander et al. used mice with complete genetic deletion of the gene encoding IL-1R1, whereas the clinical trial utilizes antibody inhibition of one of the ligands for this receptor, IL-1 $\beta$. There may be important biological differences between complete genetic abrogation of IL-1R1 signaling and partial antibody inhibition of the IL-1 $\beta$ ligand. Second, Alexander et al. used germ-line deletion of the gene encoding IL-1R1 from early prenatal development, whereas the clinical trial utilizes antibody inhibition of IL- $1 \beta$ in adults with preexisting CAD. Early loss of IL-1R1 signaling might have led to compensatory changes that could have influenced the reported phenotype. A genetic experiment in mice that comes closer to mimicking the human trial would be to induce deletion of the gene encoding IL-1R1 (or, preferably, that encoding IL-1 $\beta$ itself) in mice with established atherosclerotic lesions. Finally, while many important insights have been gained from the study of atherosclerosis in mice, there remains to this day no bona fide model of plaque rupture in mice that reproduces the pathophysiological events that underlie acute myocardial infarction and other acute coronary syndromes in humans. Thus, the field is dependent on careful histological characterization of atherosclerotic lesions in mice and comparisons with similar histologic findings in humans. For example, while the histological findings of Alexander et al. were unexpectedly interesting (21), the mice lacking IL-1R1 were not shown to have increased plaque rupture or to develop acute myocardial infarction. However, the findings are certainly provocative and hypothesis generating. They affirm the wisdom of studying three different doses of canakinumab in the CANTOS trial and suggest that
MMP3 might be an interesting biomarker to measure with regard to correlation with outcomes. More broadly, the work of Alexander et al. (21) illustrates the complexity of the interrelationships between inflammatory pathways and atherosclerosis and rebuts the notion that all inflammation is by its nature proatherogenic.

\section{Summary}

In summary, the CANTOS trial of canakinumab for inhibition of IL-1 $\beta$ in patients with CAD to test the effects of this biologic on cardiovascular events is the first direct test of the "inflammation hypothesis" in human atherosclerosis. While the study of Alexander et al. (21) has revealed additional complexities with regard to the role of IL-1 signaling in advanced atherosclerosis, the ongoing clinical trial should provide a definitive test of whether - and to what degree - interruption of IL- $1 \beta$ signaling in atherosclerosis will reduce cardiovascular events.

\section{Acknowledgments}

The author is funded by grants from the National Heart, Lung, and Blood Institute and the Leducq Foundation.

Address correspondence to: Daniel J. Rader, Perelman School of Medicine at the University of Pennsylvania, 11-125 Translational Research Center, 3400 Civic Center Blvd., Building 421, Philadelphia, Pennsylvania 19104-5158, USA. Phone: 215.573.4176; Fax: 215.573.8606; E-mail: rader@mail. med.upenn.edu.

1. Libby P, Ridker PM, Hansson GK. Progress and challenges in translating the biology of atherosclerosis. Nature. 2011;473(7347):317-325.

2. Libby P, Ridker PM, Hansson GK. Inflammation in atherosclerosis: from pathophysiology to practice. J Am Coll Cardiol. 2009;54(23):2129-2138.

3. Weber C, Noels H. Atherosclerosis: current pathogenesis and therapeutic options. Nat Med. 2011;17(11):1410-1422.

4. Dinarello CA. A clinical perspective of IL-1beta as the gatekeeper of inflammation. Eur J Immunol. 2011;41(5):1203-1217.

5. Stutz A, Golenbock DT, Latz E. Inflammasomes: too big to miss. J Clin Invest. 2009;119(12):3502-3511.

6. Schroder K, Tschopp J. The inflammasomes. Cell. 2010;140(6):821-832.

7. Henderson C, Goldbach-Mansky R. Monogenic autoinflammatory diseases: new insights into clinical aspects and pathogenesis. Curr Opin Rheumatol. 2010;22(5):567-578.

8. Walsh GM. Canakinumab for the treatment of cryopyrin-associated periodic syndromes. Drugs Today (Barc). 2009;45(10):731-735

9. Martinon F, Petrilli V, Mayor A, Tardivel A, Tschopp J. Gout-associated uric acid crystals activate the NALP3 inflammasome. Nature. 2006; 440(7081):237-241.

10. Masters SL, Latz E, O’Neill LA. The inflammasome 
in atherosclerosis and type 2 diabetes. Sci Transl Med. 2011;3(81):81ps17.

11. Larsen CM, et al. Interleukin-1-receptor antagonist in type 2 diabetes mellitus. $N$ Engl J Med 2007;356(15):1517-1526.

12. Larsen CM, Faulenbach M, Vaag A, Ehses JA, Donath MY, Mandrup-Poulsen T. Sustained effects of interleukin-1 receptor antagonist treatment in type 2 diabetes. Diabetes Care. 2009;32(9):1663-1668.

13. Fearon WF, Fearon DT. Inflammation and cardiovascular disease: role of the interleukin-1 receptor antagonist. Circulation. 2008;117(20):2577-2579.

14. Duewell P, et al. NLRP3 inflammasomes are required for atherogenesis and activated by cholesterol crystals. Nature. 2010;464(7293):1357-1361.

15. Rajamaki K, et al. Cholesterol crystals activate the NLRP3 inflammasome in human macrophages: a novel link between cholesterol metabolism and inflammation. PLoS One. 2010;5(7):e11765.

16. Hansson GK, Klareskog L. Pulling down the plug on atherosclerosis: cooling down the inflammasome. Nat Med. 2011;17(7):790-791.

17. De Nardo D, Latz E. NLRP3 inflammasomes link inflammation and metabolic disease. Trends Immunol. 2011;32(8):373-379.

18. Kirii H, et al. Lack of interleukin-1beta decreases the severity of atherosclerosis in ApoE-deficient mice. Arterioscler Thromb Vasc Biol. 2003; 23(4):656-660

19. Bhaskar V, et al. Monoclonal antibodies targeting IL-1 beta reduce biomarkers of atherosclerosis in vitro and inhibit atherosclerotic plaque formation in Apolipoprotein E-deficient mice. Atherosclerosis. 2011;216(2):313-320
20. Ridker PM, Thuren T, Zalewski A, Libby P. Interleukin-1beta inhibition and the prevention of recurrent cardiovascular events: rationale and design of the Canakinumab Anti-inflammatory Thrombosis Outcomes Study (CANTOS). Am Heart J. 2011;162(4):597-605.

21. Alexander MR, et al. Genetic inactivation of IL-1 signaling enhances atherosclerotic plaque instability and reduces outward vessel remodeling in advanced atherosclerosis in mice. J Clin Invest. 2012; 122(1):70-79.

22. Chi $\mathrm{H}$, Messas E, Levine RA, Graves DT, Amar S Interleukin-1 receptor signaling mediates atherosclerosis associated with bacterial exposure and/or a high-fat diet in a murine apolipoprotein $\mathrm{E}$ heterozygote model: pharmacotherapeutic implications. Circulation. 2004;110(12):1678-1685

\section{Redox redux: protecting the ischemic myocardium}

\section{Oded N. Spindel and Bradford C. Berk}

Department of Medicine and Department of Pharmacology and Physiology, University of Rochester School of Medicine and Dentistry, Aab Cardiovascular Research Institute, Rochester, New York, USA.

\begin{abstract}
Cardiac ischemia-reperfusion (I-R) injury occurs upon prompt restoration of blood flow to the ischemic myocardium after an acute myocardial infarction. Interestingly, many of the features of I-R injury are related to impaired mitochondrial signaling and mitochondrial dysfunction. Restoring cardiac energy bioavailability and reduction-oxidation (redox) signaling are therefore important in recovery after I-R injury. In this issue of the $J C I$, Yoshioka and colleagues describe an important and unexpected role for thioredoxin-interacting protein (TXNIP) in the control of mitochondrial respiration and cell energy metabolism. Their findings could open the door for development of TXNIP-targeted therapeutic approaches for the treatment of cardiac I-R injury.
\end{abstract}

After acute myocardial infarction, prompt restoration of blood flow to the ischemic myocardium - through the use of thrombolytic therapy or primary percutaneous coronary intervention - limits infarct size and reduces mortality. However, the restoration of blood flow can result in additional myocardial damage, a phenomenon referred to as ischemiareperfusion (I-R) injury. Despite many promising preclinical approaches to improve cardiac function after I-R, their clinical translation has thus far yielded little therapeutic benefit (1). Emerging data suggest that thioredoxin-interacting protein (TXNIP) could provide a new candidate therapeutic target $(2,3)$, and a clear rationale for this is now provided by the work of Yoshioka et al. reported in this issue of the JCI (4).

Conflict of interest: Bradford C. Berk is on the Scientific Advisory Board for Merck.

Citation for this article: J Clin Invest. 2012; 122(1):30-32. doi:10.1172/JCI61467.
TXNIP controls energy bioavailability by altering mitochondrial redox state and respiration

Many of the features of I-R injury are related to mitochondrial dysfunction manifest by the uncoupling of oxidation-phosphorylation (OXPHOS) that leads to decreased ATP production and increased ROS generation (5). Restoring cardiac energy bioavailability and homeostatic reductionoxidation (redox) signaling are therefore important in recovery from I-R. Consequently, detailed understanding of the regulation of mitochondrial homeostasis and cellular bioenergetics is key to designing therapeutic approaches to improve cardiac function after I-R.

It has recently become clear that TXNIP plays a critical role in regulating mitochondrial homeostasis and cellular bioenergetics (6-8). TXNIP is the endogenous inhibitor of the two isoforms of thioredoxin, cytosolic thioredoxin (TRX1) and mitochondrial thioredoxin (TRX2), which are key regulators of cellular redox state insert (9). Because TXNIP inhibits TRX2 in the mitochondria, and mitochondrial function is critical to recovery from I-R injury, it is logical to believe that decreasing TXNIP expression should improve mitochondrial function and recovery from I-R injury.

This hypothesis is supported by recent data showing that TXNIP has redoxdependent effects on mitochondria (Figure 1 and refs. 7,8). One set of data indicates that TXNIP-deficient mice exhibit increased glycolysis and Akt signaling associated with impaired mitochondrial fuel oxidation and loss of phosphatase and tensin homolog (PTEN) activity (7). Mechanistically, the PTEN active site contains two critical cysteine residues (Cys-71 and Cys-124) that must be in the reduced form for proper catalytic activity. Alterations in the redox state of those residues are linked to the mitochondrial respiration rate and to the ability of TRX to interact with PTEN, which is regulated by TXNIP. The second set of data indicates that TXNIP translocates from the nucleus to the mitochondria in response to changes in cellular redox state and targets TRX2 (8). As a result, TRX2 activity decreases, which induces dissociation of TRX2 from apoptosis signal regulating kinase 1 (ASK1) and release of its inhibitory effect. ASK1 dissociation from TRX2 leads to increased ASK1 activity and subsequent induction of mitochondria-mediated apoptotic signaling, including cytochrome $c$ release and caspase- 3 activation. 